\title{
Disc degeneration as a cause of low backache in young adults - MRI based study
}

\author{
Gurumurthy $B^{1}$, Purushothama Sastry ${ }^{2}$, Madhukesh Rudramurthy ${ }^{3 *}$, Venkatesh Singh ${ }^{4}$
}

\author{
${ }^{1}$ Assistant Professor, ${ }^{2}$ Professor, ${ }^{3}$ Senior Resident, ${ }^{4}$ Junior Resident, Department of Orthopedics, JSS Medical College and Hospital, \\ JSS Academy of Higher Education \& Research, Mysuru, INDIA. \\ Email: madhukesh07@gmail.com, madhukeshr@jssuni.edu.in
}

\begin{abstract}
Background: Disc degeneration includes a broad scale of clinical, radiological, and pathological manifestations. Almost $80 \%$ of population worldwide experiences low backache once in their lifetime. Disc degeneration and disc prolapse being the major contributing factors for the same. Objectives: To analyze the degree and extent of intervertebral disc degeneration in young population as a cause of low back ache. Methodology: 200 patients coming to JSSH with complaints of low back ache with a minimum duration of 3 months, underwent MRI LS spine to find the evidence of disc degeneration (Modic changes) Results: Out of the 200 patients studied , 12(6\%) patients had evidence of disc degeneration only without herniation, whereas $46(23 \%)$ patients had disc degeneration along with disc prolapse at the same spinal levels. 28(14\%) cases had disc prolapse and degeneration at different levels. 7(3.5\%) cases had no evidence of any spinal pathology. $107(53.5 \%)$ patients had disc prolapse without any disc degeneration. There was no association seen with the studied lifestyle factors. Conclusion: Disc degeneration along with disc prolapse forms the major contributing causes as low backache in young adults, hence the need for early diagnosis and management. Disc degeneration and disc prolapsed needs to be studied as separate entities for a better understanding of the disc pathology as a cause of backache in young adults.

Key Words: modic changes, MRI, disc degeneration, young adults, backache.
\end{abstract}

*Address for Correspondence:

Dr Madhukesh Rudramurthy, Senior Resident, Department of Orthopedics, JSS Medical College and Hospital, JSS Academy of Higher Education and Research, Mysuru, INDIA.

Email: madhukesh07@,gmail.com, madhukeshr@,jssuni.edu.in

Received Date: 09/01/2020 Revised Date: 28/02/2020 Accepted Date: 24/04/2020

DOI: $\underline{\text { https://doi.org/10.26611/1031431 }}$

This work is licensed under a Creative Commons Attribution-NonCommercial 4.0 International License. (cc)) EY-NC

\begin{tabular}{|l|l|}
\hline \multicolumn{2}{|c|}{ Access this article online } \\
\hline Quick Response Code: & Website: \\
& www.medpulse.in \\
& \\
\hline
\end{tabular}

\section{INTRODUCTION}

Historical texts of medicine in Greek, Arabic, Roman and Egyptian languages, dating back to 1550 B.C. , has shown detailed interests in searching for cures and management measures for various spinal diseases. ${ }^{1}$ Dating back to 200 A.D., Galen had described in detail about the pathoanatomy related to various spinal disorders and was the first person to give a detailed description on back ache ${ }^{1}$
Until $19^{\text {th }}$ century, there was no differentiation of disc degeneration from other spinal disorders .It was only after the introduction of discography in late 1940s and MRI in 1970s that there was a clear meticulous insight into the pathophysiology of disc degeneration. However, still into the $21^{\text {st }}$ century there has been no clear-cut description of the aetiology, pathology and the paramount treatment for disc degeneration. Low back pain is one of the commonest complaints that we all encounter in our clinical practice day in and out. Low back pain results in major economic consequences to the patients, in particular young employed adults, hence leading to loss of national economy indirectly, by absence from labor working days. Around 10 $\%$ of world's population suffers from low backache. Low backache is the foremost cause of disability globally, affecting around 540 million citizens, at any point of time. Around $80 \%$ of the general population develops low back pain at least one point in time. Such pain affects activities of daily living, quality of day to day life, working disability and increased health care costs.$^{2}$ In the year 
2017, Global Burden of Disease (GBD) study, Low back pain (LBP) was the top most ranked cause of the 291 conditions studied in terms of years lost to disability (YLDs), with result showing up to 83 million disability adjusted life years lost in 2017. ${ }^{2}$ Back pain results in impacting day to day activities, including sleep, of the patient. Around $40 \%$ sick leaves from workplace are because of Low backache - which is the second most common cause of workplace absenteeism, being second only to common cold.

So, a detailed study into the causes for low backache and a better understanding of the pathologies can help us encounter the problems at an earlier stage and help in prevention in their progression and the disability caused by them. Intervertebral disc degeneration in the lumbar spine is one of the major contributing factor of low back pain. The term "disc degeneration" includes a broad scale of clinical, radiological, and pathological manifestations. ${ }^{3}$ Disc degeneration is the result of gradual loss of water from spinal discs, which occurs due to natural daily stresses and minor injuries. It is due to dehydration of nucleus pulposus and increased degradation of collagen with loss of normal architecture arrangement of collagen fibres in annulus. Most of the intervertebral disc degenerations are asymptomatic, thus making finding the actual prevalence difficult. There is a recent increased incidence of intervertebral disc degeneration in young adults, presenting with backache, because of modified lifestyle pattern our generation is taking up in our day to day lives. The primary contributing factors for the disc degeneration has been previously attributed to be genetics (majority), posture, diabetes mellitus and movement patterns. Whereas, various modifiable lifestyle factors are contemplated to be involved in disc degeneration progression, which includes obesity, inactivity, smoking, alcohol, sports, repetitive activities and poor posture. But no clear association have been formed yet. This is due to limitations of previously conducted studies, such as inadequate sample volume, inconsistency in subject age, ethnicity, and radiological acquirement of data, and use of a cross-sectional study design. Intervertebral disc degeneration is the first step in the spine undergoes in the degenerative process, which is followed by the gradual progression leading to the development of osteophytes, disc space narrowing, disc prolapse, spondylolysthesis and spinal stenosis in the future. MRI (Magnetic Resonance Imaging) is the assessment gold standard for disc degeneration, with the majority of the previous studies favouring the currently preferred Modic classification. Previously conducted and published articles and standard textbook chapters on the topic of disc degeneration discussing the etio-pathogenesis consider disc prolapse alongside with disc degeneration under the same category.
This is mainly due to the lack of comprehensible definition for disc degeneration and the supposition that disc degeneration can lead to disc prolapse, these are major possible reasons for grouping these two collectively in previous research works.

Likelihood is that disc degeneration and disc prolapse are distinctive, with different etio-pathogenesis for each of them. The need for knowledge that disc degeneration and disc prolapse may be related or not related to each other is important, for the better understanding of the disc pathology. "The current study tries not only to establish the magnitude of disc degeneration leading to low back ache in young adults but also tries to highlight the pattern of degeneration seen in disc prolapse cases along with disc degeneration, in order to get a better idea about the pattern and an idea of the etio-pathogenesis of these two pathologies of the spine.

Objectives

-To analyze the degree and extent of intervertebral disc degeneration in young population as a cause of low back ache

-To study the pattern and distribution of disc degeneration in lumbar spine

\section{MATERIALS AND METHODS}

Study Design: Hospital based explorative study. Study place: JSS Medical College and Hospital, Mysuru. Study Duration: October 2017 to September 2019. Sampling technique and study population: Purposive sampling -patients coming to Department of Orthopaedics. JSS hospital in Mysore, India. Source of Data: The study will be conducted from 200 patients attending as out-patient or in-patient at Department of Orthopaedics at JSS Medical College and Hospital, Mysuru from October 2017 to September 2019 who complains of low backache, meeting the subsequently mentioned inclusion criteria.

Inclusion criteria:

- Young adults willing for Magnetic resonance imaging (MRI) evaluation of low backache

- Patients in the age group 18 to 50 years

- Patients having backpain for more than 3 months

Exclusion criteria :

1. Patients who have previously undergone surgery for spine pathology

2. Patients previously diagnosed as having back pathology other than intervertebral disc degeneration

3. Patients not willing or unable for MRI evaluation

4. Patients with spine tumors, inflammatory spinal disorders, lumbar spinal infections, spinal trauma, 
severe psychological problems, MRI sensitive implants (e.g. pacemaker), pregnancy, or having pain elsewhere more severe than low backache.

Study Design:

Hospital based Descriptive and Observational study

Sample Size:

The sample size will be the number of patients attended to at the Orthopaedics Dept of JSS medical college and hospital between October 2017 and September 2019, who meets the inclusion and exclusion criteria.

A minimum of 200 cases of each shall be included.

Study setting and Method of collection of data:

- Following approval from the Institutional Ethical Committee, Informed Consent will be taken by all the patients satisfying the inclusion criteria. Firstly, demographic data will be collected from the patient and documented

\section{METHOD}

Patients coming JSS hospital, Mysore, with complains low back ache with a minimum duration of 3 months, and meeting the aforementioned inclusion criteria are subjected to detailed history taking and general examination, followed by MRI of LS spine. Low back ache was defined as primary axial low back pain from below the costal margin to the gluteal folds, back pain that was more severe than pain in other parts of the body, every day or almost every day for at least the past 3 months. Patients are subjected to detailed history taking, regarding the duration, site, onset, character, type, radiation, relieving and exaggerating factors of pain. History regarding any previous medications taken in the past for the same complaints, recorded. Detailed history regarding any history of fever, weight loss, evening rise of temperature, cough, restriction of activities of daily living, walking distance and any other associated pain are taken to rule out any other suspected spinal pathology. History of any previous hospitalization or having any co morbidities, especially for the same complaints is asked for. Family history followed by thorough general physical examination done.

After ruling out the spinal pathologies mentioned in the exclusion criteria, we preceded with the further evaluation of the patients.

The participants are subjected to personal history assessment which included life style factors like history of smoking and drinking alcohol (at least more than once a month), occupational history and history of involvement in any sports activity in recent past.

MRI study with T1 and T2 weighted images were studied for the following parameters -
- Presence of Modic changes

- Grading of Modic changes

- Number of discs involved

- Loss of disc height \pm

- Presence of schmorl nodes

- Intervertebral disc on T2 mri hypointense/hyperintense

- Presence of disc tear / disc herniation \pm

- Nerve root compression / canal compromise \pm

- Facet arthropathy \pm

- Neural foraminal narrowing \pm

Magnetic resonance imaging (MRI) is the gold standard in diagnosing and assessing the presence of disc degeneration (DD) and its severity.

In the T2-weighted MRI sequences, water content is correlated positively with signal intensity; therefore, the healthy Nulceus pulposus (NP) is bright on T2 MRI because it contains a large amount of proteoglycan (PG) that attracts water. In conventional (T2-weighted) MR images, the NP appears bright and the AF is invisible because of its short $\mathrm{T} 2$ in the normal disc. In Disc Degeneration, PG degradation results in a secondary reduction in water content, and consequently, the NP signal darkens on T2 MRI. Therefore, the loss of MRI "signal intensity" has become synonymous with DD and is often used as a marker to relate DD with LBP.

In the current study, clinicians recognize MRI as the assessment gold standard, favoring the Modic classification and not degree Pfirrmann classification systems.

This basis for this is that as the Modic system is easy to apply, score and shows a high degree of association with degenerative change unlike the Pfirrmann system, which relies on multiple gradings, descriptors and images.

Modic changes are vertebral bone marrow MRI signal intensity changes that are attributed to various etiological determinants (external as well as internal factors)

Three types of Modic changes are identified:

Type I changes represent bone marrow edema and inflammation and appear hypointense on T1-weighted imaging (T1WI) and hyperintense on T2-weighted imaging (T2WI).

Type II changes are associated with fatty replacement of normal hemopoietic bone marrow and appear hyperintense on T1WI and isointense or slightly hyperintense on T2WI.

Type III changes represent subchondral bone sclerosis and appear hypointense on both T1WI and T2WI.

Additionally, the absence of Modic changes, indicating normal anatomical appearance, is designated as Modic type 0 
Statistical analysis: SPSS software version 21.0 was used. Chi square test along with $\mathrm{p}$ values for association of various variables used in the study

\section{RESULTS}

In the conducted the study, patients coming to JSS hospital, and meeting the inclusion criteria, and willing for the MRI evaluation of the spine for low back ache evaluation are included. The total number of patients willing for the same and underwent MRI -LS spine are 200. On the basis of MRI spinal parameter (modic changes) the patients are categorized as having disc degeneration or the absence of it. Out of the 200 MRI- LS spine studied; 58 cases are found to have some degree of disc degeneration. In the remaining 142 cases, 107 cases are found to have disc prolapsed on the studied MRI, without any evidence of disc degeneration, which formed majority of the studied cases, 7 cases have no spinal pathology per se , 28 cases have disc prolapse with associated disc degeneration at different level. Out of the 58 cases having disc degeneration, 12 cases have no associated disc prolapse, and the low back ache was attributed to be cause due to disc degeneration per sec. The remaining 46 cases have disc degeneration and disc prolapsed at the same levels in the lumbar spine. So, in total we could say that disc degeneration was contributing factor for the low back ache in $29 \%$ (58) cases. There was an association of disc prolapsed with disc degeneration in 46 cases of this group, in which the cause leading to disc prolapse itself was credited to be disc degeneration, and the former being a sequela of the latter. One category is made having only disc degeneration (12 cases) Second category having disc degeneration and disc prolapse at the same levels (46 cases). Third category having disc degeneration and disc prolapse at different levels ( 28 cases)

Table 1: Distribution of Disc Changes among the study subjects $(n=200)$

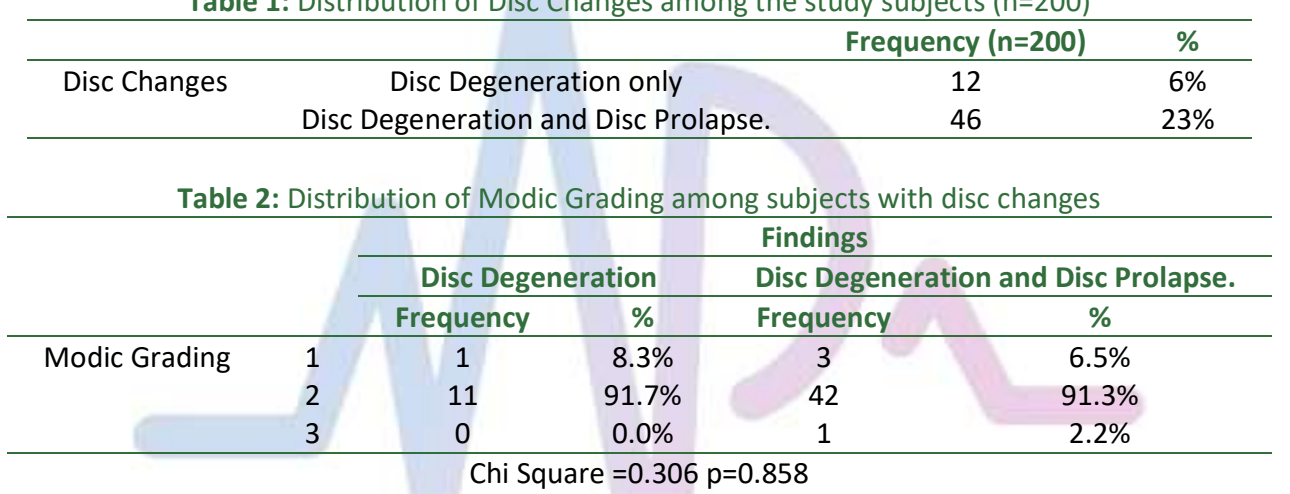

Modic changes were graded as per standardized protocols, Modic grade 2 were the most prevalent ones found in the study ( $91.7 \%$ in DD, and $91.3 \%$ in DD+DP cases), Modic type 1 were found in $8.3 \%$ and $6.5 \%$ respectively and Modic type 3 were $2.2 \%$ in second category, and none were present in the first category

Table 3: Distribution of Disc Degeneration at various levels among study subjects

\begin{tabular}{cccccc}
\hline & & \multicolumn{4}{c}{ Findings } \\
\cline { 3 - 6 } & & \multicolumn{2}{c}{ Disc Degeneration } & \multicolumn{2}{c}{ Disc Degeneration and Disc Prolapse } \\
\cline { 3 - 6 } & & Frequency & $\%$ & Frequency & $\%$ \\
\hline Disc & T12-L1 & 1 & $8.3 \%$ & 1 & $2.2 \%$ \\
Degeneration at & L1- L2 & 1 & $8.3 \%$ & 1 & $2.2 \%$ \\
Different Levels & L2-L3 & 4 & $33.3 \%$ & 2 & $4.3 \%$ \\
& L3-L4 & 7 & $58.3 \%$ & 14 & $30.4 \%$ \\
& L4- L5 & 6 & $50 \%$ & 33 & $71.7 \%$ \\
& L5-S1 & 5 & $41.6 \%$ & 25 & $54.3 \%$ \\
\hline
\end{tabular}

The first group, had disc degeneration mostly affecting the upper lumbar region, L3-L4 being most common $58.3 \%$ and L2-L3 being 33.3\%. however it was also seen in L4-L5 (50\%) and L5-S1 levels commonly as well (41.6\%)

The second group had disc degeneration along with disc prolapsed affecting the lower lumbar level mostly, $71.7 \%$ in L4L5, and second most common being L5-S1 (54.3\%). 


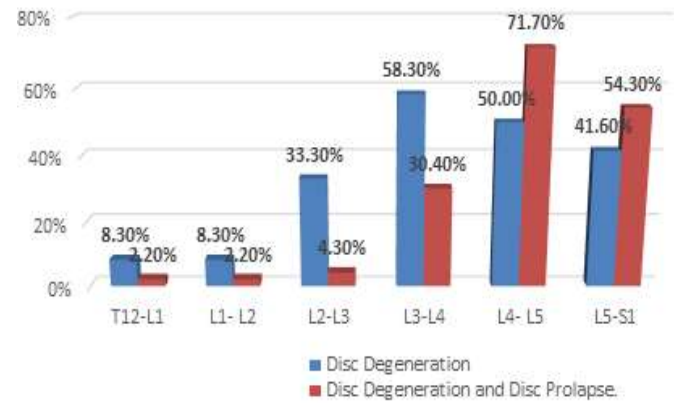

Figure 1: Disc Degeneration

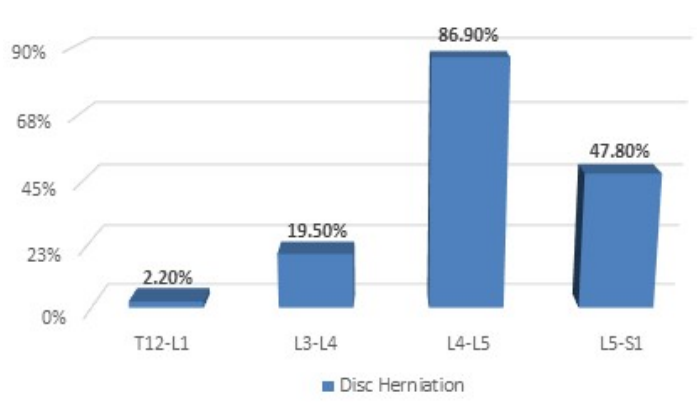

Figure 2: Loss of Disc Height

In the study, 5 cases had incidental finding of having hemangioma in lumbar spine. One case was reported to be having tarlov cyst in sacral vertebra. Spinal deformity was not seen in any of the cases.

\section{DISCUSSION}

In the current scenario, low backache is one of the foremost health problems upsetting both the citizens and the society. Back pain is robustly related with degeneration of the intervertebral disc.

The discs in the study were divided into five groups degenerated discs, degenerated discs with prolapsed discs at the same level, prolapsed discs with degeneration at different levels, and only prolapsed discs without any degeneration, lastly those with normal MRI spine.

Though disc prolapse and Disc degeneration present clinically differently, both these entities have been included as a single entity in previous discussions of disc degeneration in the past

Disc degeneration usually presents as low back pain in symptomatic patients, whereas disc prolapse manifests mainly as low back pain with or without radicular sysmptoms. Many previous studies and standard textbook chapters on disc degeneration mentioning about the etiopathologenesis and management consider disc prolapse along with disc degeneration.

Our present study tries to study these two entities as separate categories, highlighting mainly on disc degeneration leading to low back ache symptoms in the studied population. The study results clearly show the different pattern of disc degeneration seen in the different groups.

One group including disc degeneration only, being the sole cause for the low backache, affecting around $6 \%$ of the cases .The pattern of distribution of disc degeneration was found to be very different to that of the other mentioned category i.e. disc degeneration with disc prolapsed at the same level, which clearly suggests that the etio-pathogenesis for both the pathologies supposed to be different to each other, but the disc prolapse can be thought to be a sequelae of disc degeneration, which might have resulted due to weakening and giving away of the disc and end plates.
However, as we found out that the majority (53.5) of the people who met the inclusion criteria of the study were found to have only disc prolapse, without any associated disc degeneration, mostly due to mechanical straining or loading of the affected region, probably in an acute setting, with a probable positive history of strenuous activity which must have lead to the prolapsed disc .Therefore, in young adults disc prolapse is the major contributing factor for low back pain.

All the 5 discs of the lumbar spine(L1 to L5) studied had different patterns of disc degeneration involvement, indirectly suggesting that the two groups of disc degeneration and disc prolapse needs to be considered as different cohorts in any further studies to be done in future. In a similar study conducted by Rishi et al., in Ganga hospital in Tamil Nadu, in $2013,{ }^{3}$ analyzed the patterns of lumbar disc degeneration in patients with chronic back pain and disc degenerative disease (DDD) and those with acute disc prolapse(DP). DP and DDD patients differed significantly in the number, extent, and severity of degeneration. DDD patients had a significantly higher number of degenerated discs than DP patients. The incidence of multilevel and pan-lumbar degeneration was also significantly higher in DDD group. DDD patients had predominant upper lumbar involvement, whereas DP patients had mainly lower lumbar degeneration. In patients with single level DP, the majority of the other discs were non-degenerate, the lower lumbar spine was predominantly involved and the end-plate damage was higher. Patients with back pain and Disc degenerative disease had larger number of degenerate discs, early multilevel degeneration, and predominant upper lumbar degeneration. Our study also demonstrates a similar pattern of disc degeneration in these two groups, thus substantiating the findings even more and the need for a detailed analysis in further studies of these groups as separate entities.

The pattern of degenerative changes in the studied young patients, most likely indicates a systemic etio-pathology 
for the cause of disc degeneration, which might be genetic or environmental in nature. Furthermore, the absence of significant disc prolapse in these affected spinal levels suggests that different etio-pathogenic mechanisms may exist for the these two enitities, and needs to be studied in detail to avoid the already present overlapping. ${ }^{3}$

A study done on identical twins done by Jasmeet Singh Dhir et al. in 2009, supports the role of genetics in disc degeneration pathology. ${ }^{15}$

Patients with disc prolapse were present mainly at the L4L5 and L5-S1 disc levels. These regions are the most biomechanically strained segment of the spine and the lack of skipped degeneration (gap of a normal disc, between two degenerated discs) suggests that a bio-mechanical etiology plays a role in the development of disc prolapse. ${ }^{3}$ The lower lumbar spine being the most biomechanically stressed segment of the spine, the noted incidence of lower lumbar degeneration (L4-L5, L5-S1) was more (76\%) in contrast to higher lumbar degeneration (24\%) among the disc prolapse patients, further giving support to investigational biomechanical interpretations.

Whereas, on the opposite, patients having only Disc degeneration had a higher incidence of upper lumbar degeneration (54\%). Interestingly, the L1-L2, L2-L3, and L3-L4 discs, which were thought to be at a mechanically advantageous location, were more involved in the degenerative progression. We need to evaluate in future studies the orientation of the involved degenerated discs, to find out exact etiopathology of accelerated disc degeneration.

The lumbar spine section comprises of mobile segments, hence the lower spinal intervertebral discs are easily affected by mechanical and motion stresses. At the same time, the consequence of metabolic factors, such as Diabetes mellitus, on the lumbar spine disorders, may be veiled, hence it promotes us to study these factors contributing to disc degeneration, in a more comprehensive longitudinal study.

Kalliopi Alpantaki et al. conducted a study in 2019, in which review of various previous studies was done, had a result of weak clinical but strong laboratory evidence in support of diabetes being a contributing factor for disc degeneration. ${ }^{16} \mathrm{M}$. Teraguchi et al., reported a similar positive co-relation with diabetes mellitus. ${ }^{5}$

In previously conducted studies related to lifestyle factors and their association with disc degeneration, by Shivali Pankaj Gaikwad et al. in $2016^{10}$ found increased incidence of disc degeneration with increased associated with increased hours of heavy physical activity and prolonged hours of sitting. In another study done by Sudhir Ganesan et al. in $2017^{7}$ found positive association of occupation having prolonged sitting and standing with disc degeneration although there was no positive relation with respect to age,sex,smoking and alcohol consumption was made.

Our study showed highest prevalence of disc degeneration in homemakers $(41 \%$ only disc degeneration cases, $50 \%$ disc degeneration and disc prolapse cases) followed by farmers, thus suggesting frequent bending and stooping, lifting of weights, and bad posture might be contributing factors for disc degeneration.

In our study, there was no positive association of factors such as patient's age, history of smoking and alcohol intake in development of disc degeneration.

\section{CONCLUSION}

Our results urges the need to address the underlying causes of low backache, affecting majority of the country's youth, indirectly having a debilitating effect on the nation and its day to day growth $.6 \%$ of the studied population had backache which was due to disc degeneration per se. , affecting mainly the higher lumbar spinal levels $53.5 \%$ cases had disc prolapse, without any evidence of degeneration, mainly affecting the lower lumbar spinal levels. Another $26 \%$ of the patients had backache due to the disc prolapse which was a sequelae of disc degeneration at the same disc levels, affecting mainly the lower lumbar spinal levels. Hence, disc degeneration forms one of the major cause of low backache in young adults, which if diagnosed and addressed at an earlier stage can reduce the burden of low backache affecting the young individuals of the country.

\section{LIMITATIONS}

Low sample size and population under study, not clear representation of the general population, and is restricted only to hospital communit.

\section{REFERENCES}

1. Truumees E. A history of lumbar disc herniation from Hippocrates to the 1990s. Clinical Orthopaedics and Related Research ${ }^{\circ}$. 2015 Jun 1;473(6):1885-95.

2. Ravindra VM, Senglaub SS, Rattani A, Dewan MC, Härtl R, Bisson E, Park KB, Shrime MG. Degenerative lumbar spine disease: estimating global incidence and worldwide volume. Global spine journal. 2018 Dec;8(8):784-94.

3. Kanna RM, Shetty AP, Rajasekaran S. Patterns of lumbar disc degeneration are different in degenerative disc disease and disc prolapse magnetic resonance imaging analysis of 224 patients. The Spine Journal. 2014 Feb 1;14(2):300-7.

4. Teraguchi M, Yoshimura N, Hashizume H, Muraki S, Yamada H, Minamide A, Oka H, Ishimoto Y, Nagata K, Kagotani R, Takiguchi N. Prevalence and distribution of intervertebral disc degeneration over the entire spine in a population-based cohort: the Wakayama Spine Study. Osteoarthritis and cartilage. 2014 Jan 1;22(1):104-10.

5. Teraguchi M, Yoshimura N, Hashizume H, Yamada H, Oka H, Minamide A, Nagata K, Ishimoto Y, Kagotani R, Kawaguchi H, Tanaka S. Progression, incidence, and risk 
factors for intervertebral disc degeneration in a longitudinal population-based cohort: the Wakayama Spine Study. Osteoarthritis and cartilage. $2017 \mathrm{Jul}$ 1;25(7):1122-31.

6. Elliott BC, Davis JW, Khangure MS, Hardcastle P, Foster D. Disc degeneration and the young fast bowler in cricket. Clinical Biomechanics. 1993 Sep 1;8(5):227-34.

7. Ganesan S, Acharya AS, Chauhan R, Acharya S. Prevalence and risk factors for low back pain in 1,355 young adults: a cross-sectional study. Asian spine journal. 2017 Aug;11(4):610.

8. . Deane JA, McGregor AH. Current and future perspectives on lumbar degenerative disc disease: a UK survey exploring specialist multidisciplinary clinical opinion. BMJ open. 2016 Sep 1;6(9):e011075.

9. Martínez-Quiñones JV, Aso-Escario J, González-García L, Consolini F, Arregui-Calvo R. Are Modic changes able to help us in our clinical practice? A study of the Modic changes in young adults during working age. Clinical spine surgery. 2017 Jul 1;30(6):259-64.

10. Gaikwad SP, Rote-Kaginalkar V, Badarkhe-Patil P. Magnetic Resonance Imaging Lumbo Sacral Spine in Assessment of Low Back Pain in Young Adults. INTERNATIONAL JOURNAL OF SCIENTIFIC STUDY. 2016 Apr 1;4(1):25-31.

11. Jiang $X$, Chen D. Magnetic resonance imaging analysis of work-related chronic low back pain: comparisons of different lumbar disc patterns. Journal of pain research. 2018;11:2687.

12. Brinjikji W, Diehn FE, Jarvik JG, Carr CM, Kallmes DF, Murad MH, Luetmer PH. MRI findings of disc degeneration are more prevalent in adults with low back pain than in asymptomatic controls: a systematic review and meta-analysis. American Journal of Neuroradiology. 2015 Dec 1;36(12):2394-9.
13. de Schepper EI, Damen J, van Meurs JB, Ginai AZ, Popham M, Hofman A, Koes BW, Bierma-Zeinstra SM. The association between lumbar disc degeneration and low back pain: the influence of age, gender, and individual radiographic features. Spine. 2010 Mar 1;35(5):531-6.

14. Kjaer P, Leboeuf-Yde C, Korsholm L, Sorensen JS, Bendix T. Magnetic resonance imaging and low back pain in adults: a diagnostic imaging study of 40 -year-old men and women. Spine. 2005 May 15;30(10):1173-80.

15. Dhir JS, Seth R, Cheeseman C, Singh J. Identical lumbar spinal disk pathology in twins (clinical presentation and review of literature). Surgical neurology. 2009 Feb $1 ; 71(2): 250-3$.

16. Alpantaki K, Kampouroglou A, Koutserimpas C, Effraimidis G, Hadjipavlou A. Diabetes mellitus as a risk factor for intervertebral disc degeneration: a critical review. European Spine Journal. 2019 Jun 14:1-6.

17. Leboeuf-Yde C, Kjær P, Bendix T, Manniche C. Selfreported hard physical work combined with heavy smoking or overweight may result in so-called Modic changes. BMC musculoskeletal disorders. 2008 Dec;9(1):5.

18. Jackson AR, Dhawale AA, Brown MD. Association between intervertebral disc degeneration and cigarette smoking: clinical and experimental findings. JBJS reviews. 2015 Mar 31;3(3).

19. Samartzis D, Borthakur A, Belfer I, Bow C, Lotz JC, Wang HQ, Cheung KM, Carragee E, Karppinen J. Novel diagnostic and prognostic methods for disc degeneration and low back pain. The Spine Journal. 2015 Sep 1;15(9):1919-32.

20. Rothman- Simeone and Herkowitz's The Spine $7^{\text {th }}$ edition.

21. Campbell's Operative Orthopaedics , $13^{\text {th }}$ edition.

\section{Source of Support: None Declared} Conflict of Interest: None Declared

Policy for Articles with Open Access:

Authors who publish with MedPulse International Journal of Orthopedics, (Print ISSN: 2579-0889, Online) (ISSN: 2636-4638) agree to the following terms: Authors retain copyright and grant the journal right of first publication with the work simultaneously licensed under a Creative Commons Attribution License that allows others to share the work with an acknowledgement of the work's authorship and initial publication in this journal.

Authors are permitted and encouraged to post links to their work online (e.g., in institutional repositories or on their website) prior to and during the submission process, as it can lead to productive exchanges, as well as earlier and greater citation of published work. 